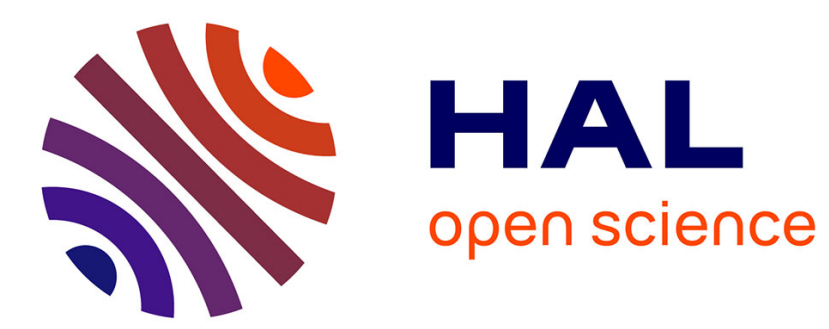

\title{
Les Sikhs dans la société indienne
}

\author{
Denis Matringe
}

\section{To cite this version:}

Denis Matringe. Les Sikhs dans la société indienne. Actes de la Recherche en Sciences Sociales, 1986, 61, pp.65-78. halshs-01956186

\section{HAL Id: halshs-01956186 https://shs.hal.science/halshs-01956186}

Submitted on 15 Dec 2018

HAL is a multi-disciplinary open access archive for the deposit and dissemination of scientific research documents, whether they are published or not. The documents may come from teaching and research institutions in France or abroad, or from public or private research centers.
L'archive ouverte pluridisciplinaire HAL, est destinée au dépôt et à la diffusion de documents scientifiques de niveau recherche, publiés ou non, émanant des établissements d'enseignement et de recherche français ou étrangers, des laboratoires publics ou privés. 


\title{
Persée
}

\author{
http://www.persee.fr
}

\section{Les Sikhs dans la société indienne}

\section{Denis Matringe}

Actes de la recherche en sciences sociales, Année 1986, Volume 61, Numéro 1

p. $65-78$

Voir l'article en ligne

Les Sikhs dans la société indienne La crise actuelle qui agite le Panjab ne peut se comprendre que si on la rapporte identité historique des Sikhs Elle résulte certes des transformations économiques démographiques et sociales qui ont affecté cette région pendant les années 1960-1970 Le développement du capitalis me agricole par les inégalités croissantes et les mouvements migratoires il entraînés boule versé le tissu social et favorisé la montée des extrémismes politiques et religieux mais sans remettre en cause et même en les intensifiant les références et les symboles de la culture sikhe selon lesquelles se retraduisent tous les change ments sociaux au Panjab

\footnotetext{
Avertissement

L'éditeur du site «PERSEE » - le Ministère de la jeunesse, de l'éducation nationale et de la recherche, Direction de l'enseignement supérieur, Sous-direction des bibliothèques et de la documentation - détient la propriété intellectuelle et les droits d'exploitation. A ce titre il est titulaire des droits d'auteur et du droit sui generis du producteur de bases de données sur ce site conformément à la loi n`98-536 du 1er juillet 1998 relative aux bases de données.
}

Les oeuvres reproduites sur le site «PERSEE » sont protégées par les dispositions générales du Code de la propriété intellectuelle.

Droits et devoirs des utilisateurs

Pour un usage strictement privé, la simple reproduction du contenu de ce site est libre.

Pour un usage scientifique ou pédagogique, à des fins de recherches, d'enseignement ou de communication excluant toute exploitation commerciale, la reproduction et la communication au public du contenu de ce site sont autorisées, sous réserve que celles-ci servent d'illustration, ne soient pas substantielles et ne soient pas expressément limitées (plans ou photographies). La mention Le Ministère de la jeunesse, de l'éducation nationale et de la recherche, Direction de l'enseignement supérieur, Sous-direction des bibliothèques et de la documentation sur chaque reproduction tirée du site est obligatoire ainsi que le nom de la revue et- lorsqu'ils sont indiqués - le nom de l'auteur et la référence du document reproduit.

Toute autre reproduction ou communication au public, intégrale ou substantielle du contenu de ce site, par quelque procédé que ce soit, de l'éditeur original de l'oeuvre, de l'auteur et de ses ayants droit.

La reproduction et l'exploitation des photographies et des plans, y compris à des fins commerciales, doivent être autorisés par l'éditeur du site, Le Ministère de la jeunesse, de l'éducation nationale et de la recherche, Direction de l'enseignement supérieur, Sous-direction des bibliothèques et de la documentation (voir http://www.sup.adc.education.fr/bib/). La source et les crédits devront toujours être mentionnés. 


\section{DENIS MATRINGE}

\section{IES SIKLS DANS}

Depuis plusieurs années déjà, les troubles du Panjab indien retiennent l'attention. Récemment, les événements se sont précipités. L'assaut donné au Temple d'Or par l'armée indienne (1), l'assassinat d'Indira Gandhi, les émeutes qui s'en sont suivies, les attentats au transistor piégé, l'anéantissement, d'un Boeing 747 en plein vol, dû probablement à des terroristes, et l'assassinat du dirigeant sikh modéré Longowal après la signature d'un pacte avec M. Rajiv Gandhi sont autant de signes d'une évolution difficile à maîtriser, dont l'issue demeure incertaine et dont les enjeux dépassent largement les frontières du Panjab et de l'Inde.

Cette éruption de violence paraît incompréhensible aux Occidentaux ; elle l'est aussi pour beaucoup d'Indiens. Ses causes profondes se laissent pourtant deviner. Les facteurs historiques y occupent une place importante : la plupart des dirigeants sikhs se réfèrent à un mythe de l'origine et du destin de leur communauté ; l'histoire en est pour eux une vaste réserve de symboles. Mais le tour pris par les événements depuis le retour au pouvoir d'Indira Gandhi et la formation d'un gouvernement congressiste dans le Pnajab en 1980 résulte aussi d'une profonde mutation économique, démographique et sociale de la société panjabie depuis le début de la «révolution verte». Prendre en compte ces facteurs historiques et ces transformations sociales permet de mieux saisir le sens des revendications avancées par les Sikhs et d'analyser avec plus de précision les derniers développements du problème panjabi .

\section{Le poids de l'histoire}

Les Sikhs ont une conscience aiguë de leur histoire et la lisent volontiers comme un double combat : pour la liberté, par la résistance à toute forme d'oppression, et contre le perpétuel danger d'une réabsorption dans l'hindouisme multiforme. Des nombreux cultes nés du renouveau hindou au

1-En Inde même, plusieurs ouvrages ont décrit, analysé et commenté l'opération «Blue Star»immédiatement après son exécution, cf. Amarjit Kaur et al., The Punjab Story, New Delhi, Roli Books International, 1984; V.-D. Chopra, US-Pak Collusion : The Punjab Theatre, in V.-D. Chopra et al., Agony of the Punjab, New Delhi, Patriot Publishers, 1984 ; Kuldip Nayar et Khushwant Singh, Tragedy of Punjab. Operation Bluestar and after, New Delhi, Vision Books, 1984 ; Sachchidanand Sinha et al., Army Action in Punjab. Prelude and Aftermath, New Delhi, Samba Era Publications, 1984.

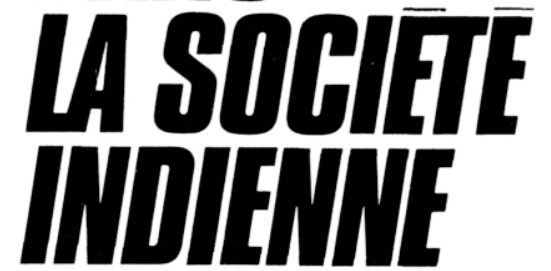

15e siècle (2), le sikhisme est en effet le seul qui soit parvenu à affirmer et à maintenir une identité autonome. Les institutions léguées par ses gourous à la communauté sikhe et la composition sociale de celle-ci y ont largement contribué.

Monothéïsme et rejet du système des castes sont au coeur de la religion prêchée par Nanak (1469-1539), le premier gourou des Sikhs, né dans la haute caste des Khattri (3). Les Sikhs résument volontiers son enseignement par un triple commandement : kirt kamao (accomplis ton travail), vand chako (partages-en le fruit), nam japo (médite sur le nom) (4). Nanak insista sur le rôle du gourou comme guide, de la communauté (sangat), du chant en congrégation des hymnes du gourou (kirtan) et du lieu de repas commun, sans distinction de caste (langar).

Ses successeurs renforcèrent encore la cohésion de la communauté par de nouvelles institutions (5). On attribue au second gourou, Angad, l'invention de l'écriture gurumukhi dans laquelle les Sikhs écrivent le panjabi et au troisième, Amar Das, la généralisation du langar, le réfectoire commun, et la première organisation territoriale et financière de la communauté, encore appelée Panth (le chemin). Le cinquième, Arjan, fit construire le Hari Mandir (le Temple d'Or d'Amritsar), renforça l'organisation économique du Panth, entreprit la conversion des paysans-guerriers jat, dont le mode de vie et les valeurs furent déterminants pour l'évolution de la communauté (6), et compila une première version de l'Adi Granth, le livre sacré des Sikhs. Il fut assassiné sur ordre de l'empereur

2-Sur les antécédents sant de la pensée religieuse de Nanak, cf. W.-H. Mc Leod, Guru Nanak and the Sikh Religion, Delhi, Bombay, Oxford University Press, 1968, pp. 151 sq. 3-L'ouvrage fondamental sur Nanak et sa religion est celui de W.-H. Mc Leod, op. cit.

4-Cf. W.-H. Mc Leod, The Way of the Sikh, Bucks, 1975, réimpr. Londres, Hulton Educational Publications, 1978.

5-Pour une présentation des dix gourous, cf. W. Owen-Cole, (avec Piara Singh Sambhi), The Sikhs. Their Religious Beliefs and Practices, Londres, 1978, réimpr. New Delhi, Rupa and Co, 1984, pp. 7 sq.

6-Cf. W.-H. Mc Leod, The Evolution of the Sikh Community. Five Essays, Delhi, Bombay, Oxford University Press, 1975 , pp. 9sq. 
Les textes qui suivent ont été publiés dans les deux principales revues panjabies de création littéraire : Arsi (Le Miroir), éditée à Delhi par Pritam Singh, et Nagmani (Le Joyau du Serpent), éditée à New Delhi par Amrita Pritam. Ils ont été écrits "à chaud» par certains des écrivains panjabis les plus lus aujourd'hui, aux temps les plus forts de la crise panjabie : avant (textes 1 et 2) et après l'opération Blue Star (textes 5 à 7), après l'assassinat d'Indira Gandhi et les massacres qui l'ont suivi (texte 8) et après l'assassinat de Longowal (texte 9). Sans entrer dans les détails, on peut signaler quelques traits saillants de ces textes pris dans leur ensemble. 1. Ils portent la marque de la stupeur et de la crainte face aux événements, le sentiment d'être dépassé et impuissant s'y mêlant aux lamentations. 2. Mais dans plusieurs textes retentissent des appels à réagir, à oeuvrer à la reconstruction de l'unité. 3. Pour que leurs plaintes et leurs appels soient entendus, les auteurs jouent des registres les plus propres à toucher leurs lecteurs : croyances religieuses (sikhisme et religion populaire) ; évocation des légendes de la tradition orale, des poèmes des soufis, des écritures sikhes et de poèmes marquants de l'époque moderne; réminiscences des heures hérö̈ques de l'histoire sikhe.

\section{«Affrontement d'idées»*}

\author{
Le premier choc \\ que j'aie vu de mes yeux \\ fut celui de deux enfants \\ qui se heurtèrent en se chamaillant pour une poupée. \\ Leurs fronts enflèrent \\ mais \\ aucun ne fronça les sourcils. \\ Le bruit de ce choc \\ ne parvint qu'à moi. \\ Le deuxième choc que j'aie vu de mes yeux \\ fut celui de deux bus \\ qui roulaient sur la route \\ dans la même direction. \\ mais s'obstinant chacun \\ à vouloir être devant l'autre, \\ si bien qu'à la fin les deux bus \\ heurtèrent les barrières de sécurité. \\ Les deux conducteurs se lançaient des injures, \\ tous deux fronçaient les sourcils. \\ Le bruit de ce choc \\ parvint aux seuls passagers. \\ Le troisième choc que j'aie vu de mes yeux \\ fut celui de l'affrontement de deux idées. \\ Sur leurs frontsluisaient des braises de haine, \\ de leurs yeux coulait du sang \\ et nues, \\ elles s'adressaient à des gens pudiques. \\ $A$ ma grande surprise \\ le bruit de ce choc, mes amis, \\ était arrivé au village \\ avant moi \\ et dans chaque maison \\ retentissaient des paroles de feu.
}

\footnotetext{
* Poème d'Ajaib Singh Hundal, Arsi, mai 1984, p. 5.
}

moghol, Jahangir, en 1606 (7), et une longue période de luttes s'ouvrit, au cours de laquelle, fort de l'apport jat, le Panth se militarisa. Le sixième gourou, Hargobind, qui siégeait en armes sur son trône, fit construire en face du Hari Mandir l'Akal Takht (Trône éternel), siège du pouvoir spirituel et temporel des Sikhs (8). Son successeur, Teg Bahadar, fut exécuté sur ordre du dernier grand Moghol, Aurangzeb, pour avoir refusé de se convertir à l'Islam. D'importants changements furent introduits dans l'organisation de la communauté par son successeur, Guru Gobind Singh, qui en paracheva la militarisation en fondant la khalsa. Ses quatre fils étant morts aux mains des Moghols, Gobind Singh, qui avait inclus dans l'Adi Granth les hymnes de son père (9), déclara qu'après lui la fonction et l'autorité du gourou passeraient dans le Granth, dès lors appelé Guru Granth Sahib, et la Khalsa. Guru Gobind Singh fut assassiné par deux Pathans en 1708.

La création de la Khalsa est traditionnellement associée au rassemblement sikh de Baisakhi 1699 à Anandpur, siège de Guru Gobind dans les collines (10). A cette occasion, le gourou, l'épée à la main, demanda de s'avancer aux sikhs qui seraient prêts à donner leur vie pour lui (cf. infra, Bhindranwale : «Nous allons mourir...»). Après un moment, un Sikh s'approcha et fut mené à la tente du gourou. Le gourou en sortit seul, son épée maculée de sang. Le manège se répéta quatre fois, après quoi les cinq volontaires, un kshatriya, un jat et trois shudra (symbole de l'égalitarisme sikh) furent montrés vivants à la foule. Des chèvres avaient été sacrifiées en leur lieu et place. Le gourou déclara que les Panj Piare (les Cinq Aimés) formaient le noyau d'une nouvelle communauté, la Khalsa («Communauté des»Purs). Les Panj Piare furent baptisés avec le nectar d' «immortalité» (amrit) remué avec l'épée à double tranchant (khand) et ils baptisèrent à leur tour le gourou. Leur baptême fut suivi de milliers d'autres. Le gourou proclama alors le nouveau code de discipline : le tabac, la viande provenant d'animaux tués selon le rite musulman et les rapports sexuels avec les musulmanes étaient désormais interdits aux membres de la Khalsa. Ces derniers se distingueraient par cinq symboles, les panj kakke (cinq «k», le nom panjabi de chacun d'entre eux commençant par la lettre $\langle\mathrm{k} »)$ : des cheveux non coupés (kesh) retenus par un peigne (kangha), une courte épée (kirpan), un bracelet de métal (kara) et une culotte courte (kacch). Les hommes ajouteraient Singh (Lion) à leur nom et les femmes, admises dans la Khalsa, Kaur (princesse) au leur. Le Panth inclurait également les Sahajdhari (littéralement "tenants de la facilité»), disciples des gourous, mais qui ne font pas leur le code de la Khalsa, gardienne de l'othodoxie.

7-Sur les conditions obscures de sa mort, cf. W.-H. Mc Leod, The Evolution of the Sikh Community. Five Essays, op. cit., p. 3. Jahangir ne pouvait tolérer un défi à son autorité politique dans une région d'une aussi grande importance politique que le Panjab (les Sikhs ne s'adressaient-ils pas à Arjan en l'appelant Saca padshah (Vrai souverain) ?, Cf. Khushwant Singh, A History of the Sikhs, Delhi, Bombay, Oxford University Press, 1977, Vol. 1, p. 50). Les extrémistes sikhs se réfèrent volontiers à cette situation, assimilant Bhindranwale à Arjan et le pouvoir central à Jahangir. 8-C'est dans l'Akal Takht que s'était retiré Bhindranwale en 1983, lui qui proclamait volontiers les Sikhs disciples de Hargobind et non d' Indira Gandhi.

9-Guru Gobind n'inclut pas ses propres hymnes dans l'Adi Granth. Ils furent, selon la tradition, rassemblés une trentaine d'années après sa mort par Bhai Mani Singh en un recueil, qui est le second livre sacré des Sikhs (mais beaucoup moins important que l'Adi Granth) : le Dasam Granth (cf. W.-H. Mc Leod (ed. et trad.), Textual Sources for the Study of Sikhism, Manchester, Manchester University Press, 1984, pp. 6sq.). 
Cet épisode est toujours intensément vivant dans l'imaginaire sikh, et Bhindranwale s'y référait fréquemment, comme il se référait souvent à l'armement de la communauté sous Guru Hargobind et Guru Gobind Singh, et les changements intervenus alors selon la tradition furent d'une importance capitale pour l'avenir de la communauté. En fait, ils résultent d'une longue évolution où se révèle l'influence des paysans guerriers jat (11) et du culte shaktique de la Devi dans les collines Shivaliks où s'étaient retirés les Sikhs, comme en atteste ce texte du Dasam Granth (12) intitulé Var Durga Ki (La Chanson de Durga) (au sens où l'on dit «La Chanson de Roland»)

10-Cf. W.-H. Mc Leod, The Evolution of the Sikh Community, op. cit., pp. 14sq.

11-Ibid., pp. 13sq. ; sur les Jat, cf. S. et H. WestphalHellbush, The Jat of Pakistan, Berlin, Duncker und Humblot, 1964 ; et Idem, Zur Geschichte und Kultur des Jat, Berlin, Duncker und Humblot, 1968

12-Dasam Granth, Shabdarath Dasam Granth Sahib (Dasam Granth édité avec notes explicatives par Bhai Ranadhir Singh), Patiala, Punjabi University, 1973, 2 vols., p. 154 .
Remémorons-nous tout d'abord l'épée à double tranchant (ici symbole de Durga) qui a créé l'univers.

Elle a suscité Brahma, Vishnu, Maheshvar (un nom Shiva) et produit les merveilles de la nature.

Elle a formé les quatorze strates (13) et donné à voir les merveilles de la nature.

Elle a mis en place les océans, les montagnes, la terre et le ciel sans support.

Elle a créé les Danavas et les Devatas (divinités) et causé leurs querelles intestines.

C'est toi, Durga, qui as donné forme aux Daityas (divinités) et les as anéantis.

C'est toi qui as donné à Rama la force de réduire Ravana en poussière.

C'est toi qui as donné à Krishna la force d'attraper Kamsa par les cheveux et de l'abattre.

Les plus grands ascètes (muni) et les Devatas, c'est toi qui les rends capables de pratiquer l'ascèse (tapas) pendant des ères (yug).

13-Etages des mondes supérieur et inférieur dans la cosmologie hindoue, cf. A.-L. Basham, La civilisation de l'Inde ancienne, Paris, Arthaud, 1976, p. 334.

\section{«En embrassant l'histoire...»*}

Une flèche acérée perce mon coeur, dans quel état est mon Panjab!

Tête baissée, chaque village-oasis est en larmes, dans quel état est mon Panjab!

Nos matins à nouveau se font noirs (1), voici qu'à nouveau l'aube s'éclipse, voici qu'à nouveau les serments sont brisés, qu'à nouveau l'on ment.

Il faut à nouveau supporter les coups de marteau et le ciseau qui s'enfonce dans le coeur.

A nouveau tombe le pollen de mes manguiers (2), pleurent les koil (3) et les branches.

Je vois à nouveau brisée l'union des frères

et se relâcher leur étreinte.

Il souffle un vent si mauvais,

je respire un air empoisonné.

Mes rivières, braves gens, sont en feu,

quels spectacles s'offrent à ma vue!

Appuyée au mur, l'âme de l'histoire pleure.

je regarde, horrifié.

Est-ce là le Panjab, braves gens,

qui fit rebrousser chemin à Alexandre (4)?

Est-ce là le Panjab, braves gens,

qui rabattit le caquet à l'Abdali (5)?

Est-ce là le Panjab, braves gens,

qui brisa la vanité de l'Européen (6) ?

Est-ce là le Panjab, braves gens,

un jotir qui ébranla le pays (7) ?

Sur cette terre coulèrent des flots de sang,

le Jallianwala Bag nous en témoigne.

Des jeunes gens marchèrent vers la potence en riant,

leur supplice nous en témoigne (8).

Ils gagnèrent le premier combat pour l'indépendance (9),

le Jardin-du-Guru, Nankana, nous en témoigne.

Si l'union se défait, l'héritage se perd.

vérité et pureté nous en témoignent.

En embrassant l'histoire, notre héritage pleure.

Braves gens, ne perdez pas la mémoire,

le serment de vos martyrs vous lie (10).

Rapprochez-vous,

oubliez vos différences, ne vous divisez pas plus avant (11)!

Reconstruisez votre destinée commune.

Les destins écrits à l'étranger, déchirez-les !

L'étranger qui est entré chez vous,

chassez-le, ne le laissez plus revenir (12)!

Les étrangers naguère avaient divisé les Sikhs,

les avaient réduits à l'esclavage.

Nous les avons vus

partir.

Mais leur jeu continue en silence,

même si ce sont des frères qui s'entre-déchirent.
C'est quand des nations se défont, braves gens,

que les frères s'entre-tuent.

Le corps doré du Panjab pourrira :

n'avalez pas une gorgée de poison, braves gens,

il se répandra dans vos veines.

Soignez le mal commun, braves gens,

réveillez-vous, résistez, le temps passe,

écrasez le serpent de la discorde?

Ecoutez mon cri de douleur, braves gens, réagissez, réagissez!

* Piara Singh Sahirai, En embrassant l'histoire, notre héritage pleure, Arsi, juin 1984, p. 6.

1 -Allusion à un autre poème fameux écrit immédiatement après la partition par le plus grand poète d'expression ourdoue depuis Iqbal, Faiz Ahmad Faiz, un Panjabi (Faiz Ahmad Faiz, Dast-e saba (La main du Zéphyr), Lahore, 1952, pp. 112sq.; traduction française par D. Matringe in Bulletin d'études indiennes, 3, 1985.

2-Nombre d'intellectuels panjabis (tout particulièrement des écrivains), de part et d'autre de la frontière, considèrent que la partition, en séparant les communautés, en mettant fin à de précieux contacts et à une émulation enrichissante, a eu un effet mutilant et stérilisant sur la création littéraire dans le Panjab.

3-Les kokila- de la poésie sanskrite, coucous indiens renommés pour leur chant (Eudynamis orientalis).

4-Allusion à la brève incursion d'Alexandre-le-Grand dans le Panjab en 326 av. NE.

5-Souverain afghan qui envahit 9 fois le Panjab entre 1748 et 1767. 6-Allusion à la conquête de l'indépendance.

7-Allusion à l'activité des membres du Ghadr Party ou au rôle joué par les Panjabis dans le mouvement national au début des années 1920 .

8-Allusion, entre autres, à Bhagat Singh.

9-A savoir, selon cette vue commune chez les Sikhs, la libération des gurdvara.

10 - La notion de martyr (sahid) est très importante dans la religion sikhe depuis la mort de trois des dix premiers gourous aux mains des Musulmans. C'est ainsi que, d'une certaine façon, la mort "en martyr» de Bhindranwale a considérablement grandi son image. Piara Singh Sahirai (Le malheur du Panjab, Arsi, juillet 1984, pp. 3 sq.), l'auteur du poème, écrivait au lendemain de l'opération Blue Star : «Bhindranwale n'était qu'un personnage comme il en surgit régulierement dans notre histoire. L'action de l'armée l'a élevé au rang d'un martyr. Tous les Sikhs de bon sens qui jusqu'à présent étaient opposés à la ligne de Bhindranwale, à la violence, au terrorisme et au séparatisme, sont aujourd'hui cruellement blessés et voient en lui un martyr».

11 -Cf. le poème qui suit : «Nous quatre vieux amis».

12-Allusions à «la main de l'étranger» constamment évoquée par le gouvernement central et le parti du Congrès à propos de la crise panjabie. C'est bien entendu le Pakistan et, à travers lui, les U.S.A. qui sont visés. 


\section{"Nous quatre vieux amis»*}

Hindous, Musulmans, Sikhs, Chrétiens,

Tous quatre frères nés d'une même mère,

Dieu sait pourquoi nous nous soupconnons,

Adultes devenus. Nous sommes quatre vieux amis.

L'un fait l'offrande, l'autre la prière,

Dans chaque maison l'on se réjouit,

C'est le même Dieu qu'honorent

Brahmanes et mullas. Nous sommes quatre vieux amis

C'est toi qui as raison et moi tort,

Nos coeurs ne sont pas divisés,

A cause de broutilles,

Pourquoi nous détester. Nous sommes quatre vieux amis.

Nos yeux ont faim d'amour.

Renonçons aux propos cruels,

$\mathrm{Ne}$ vois-tu pas? C'est dans le sens du vent

Que vont les sages gens. Nous sommes quatre vieux amis.

Tout est encore possible,

Le cours de la violence peut être arrêté,

Si pour construire un barrage

S'unissent les bons nautonniers. Nous sommes quatre vieux

amis.

Villageois, citadins, propriétaires, artisans,

Si tombent les barrières inutiles,

Les voici s'embrassant,

Oubliées leurs dissensions! Nous sommes quatre vieux amis.

$N$ 'écoutons point ceux qui veulent diviser, séparer,

Mais clouons-leur le bec,

Apprenons à vivre, et de guirlandes de confiance

Jonchons les rues! Nous sommes quatre vieux amis.

* Le poème, En embrassant l'histoire, qui précède fait écho à ce poème fameux publié, aux temps des émeutes communalistes qui précédèrent la partition, par le plus grand poète Panjabi hindou, Dhani Ram Catrik, Nous quatre vieux amis, in Catrik racnavali (Oeuvres complètes de Catrik), vol. 1, ed. S. S. Amol, Patiala, Punjabi University, 1976, p. 1182.
Les années 1708-1799 furent celles où les Sikhs eurent à combattre à la fois contre les gouverneurs moghols et les envahisseurs afghans, mais finirent par se rendre maîtres du Panjab (14).

La prise du pouvoir par les Sikhs a été évoquée par Varis Shah (15), auteur du plus beau poème épico-lyrique de la littérature panjabie, en ces vers :

Maintenant que les Jat sont devenus les maîtres du pays, un nouveau pouvoir prévaut en tout lieu.

Les nobles sont mal en point, les vilains prospères, c'est un grand printemps pour les zamindar (sens à l'époque : "cultivateurs indépendants»).

Les voleurs (c'est-à-dire les Sikhs des misal ; c'est un musulman qui parle !) sont devenus de respectables chefs de village, mon ami, et il y a quatre fois plus de mauvais esprits (toujours les Sikhs ) qu'auparavant.

Jusqu'en 1716, sous la direction de Banda Bahadar, ils propagèrent un mouvement de révoltes paysannes contre le pouvoir moghol et ses institutions. Les Sikhs furent ensuite réprimés et décimés par les Moghols, puis par les Afghans. Mais dans la confusion qui régnait alors dans le Panjab, ils se réorganisèrent et parvinrent à établir un pouvoir territorial et politique, d'abord sous la forme d'une confédération de misal (groupes «égaux» de Combattants), puis dans un Etat indépendant qui vit le jour sous l'égide de Ranjit Singh, Maharajah du Panjab de 1799 à 1839 (16).

Sous Ranjit Singh et au cours des dix années (17) de querelles de palais et d'anarchie qui suivirent sa disparition, l'orthodoxie religieuse se relâcha à tel point que la réabsorption du sikhisme dans l'hindouisme semblait inéluctable. Mais la politique des Britanniques après l'annexion du Panjab en 1849 et la position de petite minorité des Sikhs dans le Raj en décidèrent autrement. En effet, d'une part, leur loyauté lors de la révolte des cipayes en 1857 valut aux Sikhs un recrutement préférentiel dans l'armée, où les Britanniques leur demandèrent d'observer les symboles et le code de la Khalsa. D'autre part, leurs qualités d'agriculteurs firent d'eux les principaux bénéficiaires de l'ouverture des Canal Colonies à partir de 1880. Enfin, l'activité des missionnaires chrétiens et des propagandistes hindous de l'Arya Samaj poussa l'élite urbaine des Sikhs à s'organiser dans la Singh Sabha (Société des Lions) (18). Les Sabhaïtes, tout en

14-On verra plus loin l'importance symbolique de cette époque. Pour un commode aperçu d'ensemble de l'histoire des Sikhs, cf. Khushwant Singh, A History of the Sikhs, $o p$. cit., vol. 1 et 2 . Pour une histoire détaillée des Sikhs au $18 \mathrm{e}$ siècle, cf. Hari Ram Gupta, History of the Sikhs, New Delhi, Munshiram Manoharlal, 1978-1982, vol. 2-4.

15-Varis Shah, Hir, Abdul Aziz (ed.), Lahore, Punjabi Abdi Academy, 1964 ; traduction française par D. Matringe, Hir Varis Shah. Introduction, translittération, traduction et commentaire, vol. 1, à paraître aux Publications de l'Institut français d'indologie de Pondichéry.

16-Sur cette période, cf. Gulshan Lal Chopra, The Punjab as a Sovreign State (1799-1839), Lahore, 1929, réimpr. Lahore, Al-Biruni, 1979 ; Khushwant Singh, Ranjit Singh Maharajah of the Punjab 1780-1839, Bombay, George Allen and Unwin (India), 1962.

17-Sur cette période, cf. Barkat Rai Chopra, Kingdom of the Punjab (1839-1845), Hoshiarpur, U.U.R. Institute, 1969. 
restant fidèles au Raj, s'attachèrent à développer les institutions, les cérémonies et les publications sikhes.

Très vite, les Sikhs eurent l'occasion d'affirmer leur identité dans leur confrontation croissante avec les Britanniques. Certains épisodes sont restés de vivants symboles. Dès la fin des années 1860 , les Sikhs de la secte des Kukas avaient fomenté des troubles, alors sévèrement réprimés. Au cours des premières décennies du 20e siècle apparurent les premiers signes d'agitation : en 1908, contre la mainmise gouvernementale sur le Managing Committee du Khalsa College d'Amritsar ; la même année, contre l'interdiction du port du turban dans l'Indian Medical Service et les cours de justice londoniennes. En 1909, les Sikhs manifestèrent pour la reconnaissance du mariage sikh. Dans les années 1910, des émigrés sikhs de la côte ouest du continent nord-américain organisèrent le Ghadr Party (Parti de la Révolte) dont le but ultime était la libération de l'Inde. Quelques milliers de militants s'infiltrèrent dans le Panjab où fut distribuée une grande quantité de littérature «séditieuse». Il y eut même une tentative de débarquement. Le mouvement fut démantelé par les autorités britanniques. Vint la Première guerre mondiale. Les Sikhs avaient espéré que leur contribution à l'effort de guerre, proportionnellement la plus importante de l'Inde, leur vaudrait, comme minorité, des clauses de sauvegarde particulières. $\mathrm{Au}$ lieu de cela, le gouvernement refusa de protester contre les discriminations dont étaient victimes les émigrants sikhs au Canada et aux USA. Le Government of India Act de 1919 n'accorda pas aux Sikhs la représentation préférentielle qu'ils avaient espérée au Punjab Legislative Council. Les Sikhs ne furent pas épargnés par la répression consécutive aux mesures Rowlatt de «justice» expéditive contre l'agitation politique et, en avril 1919, le général Dyer fit procéder dans le Jallianwala Bag d'Amritsar à un véritable massacre des participants à un rassemblement non violent. C'est dans cette atmosphère que les Sikhs entreprirent de ravir la gestion administrative, financière et religieuse des gurdvara, leurs lieux de culte, aux mahant, officiants à demi hindouisés et corrompus, maîtres des lieux depuis les troubles $\mathrm{du} 18 \mathrm{e}$ siècle. Le gouvernement prit position en faveur des mahant. Les Sikhs réagirent en formant le Siromani Gurdvara Prabandhak Kameti (SGPC) (Comité Central d'Administration des Gurdvara) en 1920, et en s'organisant dans l'Akali Dal (l'Armée des Immortels) en 1921. Les Akalis subirent une répression sanglante de la part du gouvernement et des mahant. La mort d'une centaine d'Akalis aux mains de bandits thagg payés par les mahant à Nankana Sahib, le lieu de naissance de Guru Nanak, est encore dans toutes les mémoires. Mais l'Akali Dal eut finalement gain de cause. Le contrôle des gurdvara fut transféré au SGPC aux termes

18-Sur cette question, cf. D. Matringe, L'apparition de la nouvelle et du roman en panjabi (1930-1947), Journal asiatique, 3-4, 1985. Le développement qui suit reprend en partie cet article. Sur l'importance symbolique des événements qui y sont rapportés dans la conscience sikhe, cf. les poèmes de Piara Singh Sahirai.

\section{«J'invoque Varis Shah»*}

J'invoque aujourd'hui Varis Shah (1) : «Parle, de n'importe où, de ta tombe!

Et du livre de l'amour aujourd 'hui tourne encore une page ! Une fille avait pleuré, une enfant du Panjab, et tu écrivis une romance.

Les filles sont aujourd'hui des milliers à pleurer, qui te disent :

'Lève-toi, sympathisant des malheureux ! Lève-toi, regarde ton Panjab!

Le marais (des amours de Hir et Ranjha, héros du poème de Varis) est aujourd'hui jonché de cadavres et pleine de sang la Canab (rivière de leur première rencontre).

Quelqu'un aux cinq rivières a mêlé du poison,

Et la terre a été arrosée de leur eau.

Du poison a germé dans chaque parcelle de cette terre fertile,

Qui s'est un peu partout couverte de taches rouges et de calamités.

Un vent vénéneux alors a soufflé sur les forêts,

De chaque flûte en bambou qui a fait un serpent,

Et voici que les serpents ont hypnotisé les gens et mordu, mordu.

En tout lieu, le corps du Panjab a bleui.

Les chants ont rompu avec les gorges, les fils avec les fuseaux, Les compagnes avec les parties de filage, les rouets se sont tus.

Luddan ( le batelier de la légende de Hir) a fait couler le bateau lit (où se sont rencontrés les deux jeunes gens), La balançoire (de Hir enfant) a brisé les branches du pipal. Elle est perdue, cette flûte (celle de Ranjha) où chantait le souffle de l'amour,

Et les frères de Ranjha ont tous oublié son jeu.

Le sang s'est épanché sur le sol, il s'écoule des tombes.

Les princesses de l'amour aujourd hui pleurent dans les sanctuaires.

Tous aujourd'hui sont devenus des Kaido (l'oncle malfaisant de Hir), voleurs d'amour et de beauté.

Où trouver aujourd'hui un autre Varis Shah??

J'invoque aujourd'hui Varis Shah :«Parle, de n'importe où, de ta tombe?

Et du livre de l'amour aujourd 'hui tourne encore une page !»

*Le texte intitulé «En embrassant l'histoire», écrit peu avant l'assaut du Temple d'Or, et le suivant, «Mon Panjab-fleur», écrit immédiatement après, sont pleins du souvenir de ce poème fameux écrit par Amrita Pritam lors de la partition du Panjab et repris dans Amrita Pritam, Mai jamha tu (Ensemble toi et moi), New Delhi, Nagmani, 1977 (traduction française par Denis Matringe, Hir Varis Shah à paraître aux publications de l'Institut français d'indologie de Pondichéry).

$1-$ Grand poète panjabi du $18 \mathrm{e}$ siècle. 


\section{«Mon Panjab-fleur»*}

\section{De cette boite ouverte de Pandore}

sont sortis des pythons et autres serpents sans nombre, sont sortis toutes sortes de scorpions et de guêpes, sont sortis des dragons crachant le feu.

Ils ont empoisonné la vie de leurs morsures, ces êtres venimeux surgis innombrables. Ils se prosternent devant une déesse noire aux traits d'ogresse.

Des harpies sont descendues sur terre, assoiffées du sang des hommes.

Des Raksha (1) flairent les hommes, mettent fin aux jeux enfantins.

Les sorcières (2) aujourd'hui n'épargnent aucun foyer, coupant les habits d'une façon nouvelle. Elles interrompent le cours de la vie, les vivants redoutent leurs terribles regards.

Un Yama (3), une puissance mauvaise venue de quelque enfer s'est emparée de mon Panjab.

Le ciel bleu aujourd'hui de noir s'est voilé, pas une étoile aujourd'hui ne sourit.

Au lieu d'eau pure, du ciel aujourd'hui

s'est abattue une pluie de poison.

$L$ 'eau s'est évaporée de mon Panjab-fleur,

il y coule du sang bleu, les serpents l'ont mordu.

Des Raksha et des Shakti (4) allument des bûchers, des esprits et des revenants (5) règnent en maîtres, je vous

\section{Le pays entier est horrifié}

de son propre désordre, je vous le dis.

Les plantes ne restent pas vertes,

les champs sont incendiés, je vous le dis.

Les maisons sont détruites, braves gens,

comme on abattrait des murs de sable, je vous le dis.

Un sort aujourd'hui a été jeté à mon Panjab,

ses oiseaux ont tu leur doux chant.

La brise ne souffle plus sur les jardins,

les paons ne lancent plus leurs appels.

A ujourd'hui le printemps ferme les yeux,

la ronde des saisons ferme sa porte.

Ceux qui sont pris à ce cycle infernal n'y peuvent rien.

n'ont plus qu'un destin dérisoire, sont pris au piège.

Où que vous retrouviez le fil de l'humanité,

saisissez-le, ne le lâchez plus, braves gens!

$S$ 'il apparaît en bordure des nuages noirs,

attrapez le pan de l'espoir, vous qu'on assassine, braves gens !

Vous combattez pour la justice et le droit (6),

reconnaissez-vous, braves gens!

Sachez à nouveau qui vous êtes, mes frères,

et conformez votre destin à votre héritage, braves gens !

Vous les guerriers de la lumière (7),

debout, combattez à nouveau les ténèbres!
Quelles que soient vos peines, elles s'envoleront,

notre aurore à nouveau montrera son visage rayonnant.

On rira sans éclipse,

des fleurs remplaceront les larmes de deuil.

La splendeur de l'unité sera éclatante,

on ne parlera plus des tiens ni des miens.

Vous avez le même sang,

écoutez l'appel de votre pieuse mère, braves gens!

Songez aux martyrs du Jallianwala Bag,

au sacrifice des Kukas, braves gens!

Le communalisme est un poison,

dont l'amour est l'antidote, braves gens!

Puissent les corps guérir, les âmes être purifiées

et resplendir à nouveau l'unité, braves gens !

* Piara Singh Sahirai, L'eau s'est évaporée de mon Panjab-fleur, Arsi, août 1985, p. 14.Cf. Khushwant Singh, Ranjit Singh Maharajah, $o p$. cit. Ce poème est écrit en bait-chand, le vers des poèmes épiques et épico-lyriques de la tradition panjabie classique (traduction française de Denis Matringe, Hir Varis Shah, vol. 1, à paraître aux publications de l'Institut français d'indologie de Pondichéry).

1 -Nom d'une classe de démons de la mythologie hindoue. Cette évocation de démons hindous, de sorcières, n'est pas un pur ornement poétique, mais correspond à la réalité vécue par nombre de villageois sikhs du Panjab, dont l'univers surnaturel est peuplé d'êtres empruntés à l'hindouisme et à la religion populaire (Cf. H. A. Rose, A Glossary of Tribes and Castes of the Punjab and North-West Frontier Province, Lahore, 1883, réimpr. Patiala, Languages Department Panjab, 1970, pp. 61sq.).

2-Cf. Khushwant Singh, Ranjit Singh Maharajah, op. cit., et D. Matringe, Hir Varis Shah, op. cit., à paraître $(60$ d.) : "Les sorcières sont censées poursuivre les vivants, jeter le mauvais oeil et faire des plus faibles leurs victimes. On leur impute la responsabilité de toutes sortes de maladies. En apprenant un charme de deux lettres et demie, n'importe qu'elle femme peut devenir sorcière».

3-Dieu de la mort dans l'hindouisme.

4-Cf. Khushwant Singh, Ranjit Singh, op. cit.. La shakti est la "force active d'un Dieu personnifiée dans la partie féminine de sa double nature» (N. Stchoupak, L. Nitti, L. Renou, Dictionnaire sanskrit-français, Paris, A. Maisonneuve, 1932, rééd. 1980 ), notamment celle de Shiva, auquel cas elle revêt souvent l'aspect terrible du Durga.

5-Les paysans panjabis sont constamment en butte aux «esprits». Les plus redoutables sont «ceux des hommes dont les rites funéraires n'ont pu être accomplis. Ils tourmentent les vivants pour obtenir d'eux ces cérémonies, ainsi que des rituels d'apaisement» (Cf. D. Matringe, Hir Varis Sah, op. cit., à paraître, $60 \mathrm{~d}$.).

6-Mais Bhindranwale aussi tenait ce genre de propos à ses fidèles.

7-Le ton ici est celui des poèmes qu'écrivaient les révolutionnaires ghadrites, cf. les vers suivants, cités par N.-G. Barrier, The Sikhs and their Literature, Delhi, Manohar Book Service, 1970, p. 106

«Levez-vous, fils de l'Inde chérie ! Pourquoi avez-vous dormi si longtemps?

Vous avez dormi au clair de lune, mais regardez ! Maintenant le soleil est levé». du Sikh Gurdwaras Act de 1925 (19). De nos jours encore, le SGPC, démocratiquement élu et contrôlé jusqu'ici par l'Akali Dal, joue un rôle important dans les affaires sikhes. A la même époque, les Sịkhs étaient entrés en masse dans le mouvement pour l'indépendance, auquel ils fournirent un éminent martyr en la personne de Bhagat Singh, jeune activiste de gauche pendu à Lahore pour terrorisme en 1931. Les Sikhs condamnèrent le Communal Award de 1932 et le Government of India Act de 1935 qui assuraient aux Musulmans, par le système des électorats séparés et de la

19-L'une des revendications de l'Akali Dal aujourd'hui est l'attribution au SGPC du contrôle de tous les gurdavara de l'Inde. (Le livre fondamental sur l'histoire de l'Akali Dal est celui de A.-S. Narang, Storm over the Sutlej. The Akali Politics, Delhi, Gitanjali Publishing House, 1983). représentation préférentielle des minorités, un rôle déterminant pour l'avenir du Panjab. Après que les Musulmans eurent demandé la création du Pakistan en 1940, les Sikhs tentèrent jusqu'au bout, aux côtés du Congrès, de s'opposer à la partition. Quand il fut acquis que la division du Panjab ne pourrait être évitée, le représentant akali soumit à la Cabinet Commission, en 1946, un projet d'Etat sikh indépendant (première mouture du Khalistan) qui ne fut même pas pris en considération, faute d'une zone où les Sikhs fussent en majorité.

La partition, entraînant l'installation dans le Nord-Ouest du Panjab indien de plus de 2,5 millions de réfugiés sikhs, changea cette situation. L'Inde indépendante étant un Etat laïque, ce fut au nom des frontières linguistiques que l'Akali Dal demanda la formation d'un Etat panjabi (Panjabi Suba) (20) 
qui comprît les districts de langue panjabie du PEPSU (Patiala and Eastern Punjab States Union, les anciens Etats princiers du Panjab britannique). $\mathrm{Au}$ terme d'une longue agitation politique qui aboutit, précédent mémorable, à l'investigation du Temple d'Or par la police en 1955, les Akalis eurent gain de cause. En 1956, le PEPSU fut rattaché au Panjab et après une nouvelle campagne d'agitation, en 1966 le nouvel Etat fut divisé entre un Panjab à majorité sikhe et de langue panjabie et les Etats de Haryana et Himachal Pradesh, à majorité hindoue et de langue hindie.

Les difficultés apparurent rapidement. Des. enclaves linguistiques se trouvaient du mauvais côté des nouvelles frontières (21), et le Punjab Reorganization Act, au grand dam des Akalis, faisait de Chandigarh la capitale commune du Panjab et du Haryana et plaçait le système des eaux et le barrage de Bhakra sous le contrôle du gouvernement central. Par ailleurs, des voix s'élevèrent bientôt à l'intérieur du camp akali pour demander plus d'autonomie pour le Panjab, voire même, pour certains, l'indépendance pure et simple, au nom des intérêts supérieurs de la «nation sikhe».

Depuis 1966, la situation a évolué en fonction des résultats électoraux (cf. tableau 1). Quand

Tableau 1-Les élections locales dans le Panjab

\begin{tabular}{|c|c|c|c|c|c|}
\hline & 1967 & 1972 & 1977 & 1980 & 1985 \\
\hline ongrès & 37,6 & 42,84 & 34,07 & 48 & 37,7 \\
\hline al (modérés) & 20,46 & & & & \\
\hline kali Dal (radicaux) & 4,23 & 27,65 & 31,41 & 26,90 & 38 \\
\hline $\begin{array}{l}\text { CI (parti communiste } \\
\text { nciennement pro-URSS }\end{array}$ & 5,16 & 6,65 & 6,10 & 6,50 & ? \\
\hline $\begin{array}{l}\mathrm{CI} \text { (marxiste) (PC } \\
\text { nciennement pro-chinois }\end{array}$ & 5) 3,26 & 3,15 & 3,50 & 4,10 & ? \\
\hline $\begin{array}{l}\text { ana Sangh (parti hindou } \\
\text { e centre - droit) }\end{array}$ & 9,85 & 4,97 & & & ? \\
\hline $\begin{array}{l}\text { Janata (coalition } \\
\text { de partis de droite) }\end{array}$ & & & 14,99 & 4,70 & ? \\
\hline $\begin{array}{l}\text { haratya Janata } \\
\text { roite hindoue) }\end{array}$ & & & & 5,40 & \\
\hline dépendants et al. & $\begin{array}{l}19,44 \\
00\end{array}$ & $\frac{14,74}{100}$ & $\begin{array}{c}9,93 \\
100\end{array}$ & $\begin{array}{c}4,40 \\
100\end{array}$ & \\
\hline
\end{tabular}

Source : A. S. Narang, Storm over the Sutlej, op. cit., pp. $180,191,193$ et p. 179 pour les résultats en nombre de sièges pour 1985.

20-Conçu par les extrémistes comme une étape décisive sur le chemin du Khalistan. Le dirigeant akali radical Master Tara Singh avait lui-même déclaré en 1961 (The Spokesman Weekly, 9 juillet 1961, cité par A.-S. Narang, op. cit., p. 93) : "The Hindus have got Hindustan, the Muslims have got Pakistan, what have the Sikhs got ?». 21-D’une façon générale, la «question linguistique» dans le Panjab a toujours été épineuse, chaque communauté défendant âprement le statut et l'usage de sa langue de culture : les Musulmans, ceux de l'ourdou (avant 1947), les Hindous, ceux du hindi et les Sikhs ceux du panjabi (cf. P. Brass, Language, Religion and Politics in North India, Berkeley, Cambridge University Press, 1974, pp. 277sq). Jusqu'en 1947, le Panjab avait pour langue officielle, outre l'anglais, le persan dans ses districts de l'ouest et l'ourdou dans ceux de l'est, remplacés après l'indépendance par le hindi. Pour conforter cet état de fait, à l'appel de plusieurs de leurs organisations, nombre de Panjabis hindous avaient déclaré, lors du recensement de 1951, le hindi -que certains savaient à peine-comme leur langue maternelle (cf. A.-S. Narang, op. cit., p. 96). D'où cette remarque amère d'un Akali: "While others got states for their languages, we even lost our language» (cité par P. Brass, op. cit. p. 320).

\section{"L'arrogance des armées»*}

Les armées, de quel pays viennent-elles?

De quelle ville apportent-elles le poison, de qui la fureur.

de quel cobra le souffle

qui renverse les murs,

qui mord le Sifat-sarovar (1),

qui met le feu aux pierres,

dans le Harimandar qui déverse

des torrents de venin?

Les armées, de quel pays viennent-elles?

Ayant reçu ce présent nous errons,

toi et moi comme des folles,

à nous déchirer le front.

Des traditions gravées

par les siècles dans nos mémoires,

en nos coeurs innées,

nous sont par la force aliénées.

Les armées, de quels pays viennent-elles?

On raille au loin notre pays,

des frères des belles-soeurs

font subir à leur Hir (2)

ces humiliations.

Montée (3) sur le toit,

j'appelle au secours ;

dans un coin de ma maison,

on égorge des gens.

Les armées, de quel pays viennent-elles?

Les armées viennent, les armées s'en vont,

les empires s'effondrent ;

pourrons-nous demain

revivre en voisins?

Bien que proches, souffrirons-nous

les affres de la séparation?

O l'arrogance des armées,

poussées par la haine.

Les armées, de quel pays viennent-elles?

* Poème de Haribhajan Singh, Arsi, août 1984, p. 4.

1-Le bassin du Temple dOr (littéralement le «Bassin de la Glorification").

2-Héroïne de la légende de la tradition orale la plus chère au coeur de tous les Panjabis (cf. le poème d'Amrita Pritam, cité ci-dessus).

3-Le fait que Haribhajan Singh, comme dans le texte suivant Kartar Singh Duggal, parle au féminin évoque la tradition de la poésie soufie du Panjab, dans laquelle le poete s'identifie à l'une des héroïnes des légendes de la tradition orale. Cette héroïne figure l'ầme du poète en quête d'union mystique avec Dieu, symbolisé par le héros de la légende (Cf. D. Matringe, Hir Varis Shah,op. cit., paragr. 9). 


\section{"Ne pouvoir vivre ni mourir»*}

Daz-daz, thus-thus,

thah-thah, tar-tar,

de ci, de là.

de droite, de gauche,

combien de temps encore exploserez-vous,

combien de temps encore tomberez-vous?

Ce sont des balles,

pas de la grêle

ni des gouttes de pluie.

Je le demande,

ces bombes,

ces obus, d'où viennent-ils?

Ces fusils, ces carabines,

qui les donne

au Royaume-de-la-Vérité (1)?

Où habite Dieu,

où se font le jour et la nuit

n'est de pluie que l'Amrit (2).

Qui sont-ils?

Des nôtres, des étrangers?

Ils font du vacarme,

ne se lavent pas les pieds,

ne retirent pas leurs bottes (3).

Au fil de ma promenade,

mon coeur tressaille.

Que d'obus lancent chars et blindés !

On tue,

on meurt.

Qui sont-ils, d'où viennent-ils?

Et maintenant, jusqu'à quand dureront ces

daz-daz, thus-thus,

thah-thah, tar-tar?

Je suis blessée,

je suis écorchée.

Quelqu'un dit:

«C'est cela le sikhisme!»

l'autre dit

«la panjabité».

On pille,

on saccage.

Moi, je suis l'Amour,

tison rougeoyant.

Etablie dans le temple de la Vérité et de la Non-Violence (4) à ne pouvoir vivre

ni mourir.

* Poème de Kartar Singh Duggal, Arsi, août 1984, p. 5.

1-La figure sikhe des cinq «royaumes» (khand) qui jalonnent l'élévation vers Dieu de l'âme purifiée remonte à Guru Nanak.

Le Sacc Khand (Royaume de la Vérité) est le royaume ultime, celui où l'âme s'unit à Dieu et échappe à la transmigration (Cf. W.-H. Mc Leod, Guru Nanak and the Sikh Religion, op. cit., pp. 224sq.)

2-«Nectar d'immortalité» préparé à base d'eau sucrée» (Cf. W. Owen-Cole, op. cit., p. 19), utilisé dans la cérémonie du baptême depuis la fondation de la Khalsa.

3-Gestes d'humilité et de respect à accomplir avant d'entrer dans le Temple d'Or.

4-La Vérité représente ici le sikhisme (elle y est l'un des noms de Dieu), et la Non-Violence l'hindouisme.
l'Akali Dal a été à la direction des affaires à la tête de coalitions anti-Congrès, après ses succès de 1967 et 1977, il s'est montré modéré dans ses exigences et ses manifestations et capable de contrôler son aile radicale. Par contre, quand au lendemain de la guerre victorieuse contre le Pakistan en 1971 et dans la foulée du retour d'Indira Gandhi en 1980 le Congrès a gagné les élections de 1972 et 1980 dans le Panjab, l'Akali Dal a subi l'influence de ses radicaux et des groupes extrémistes, et il a mené de vastes campagnes d'agitation. Celles-ci ont pour point d'ancrage depuis 1973 des revendications exprimées, pour la plupart, dans l'Anandpur Sahib Resolution (22). Les Akalis réclament un statut d'autonomie pour le Panjab, le rattachement de Chandigarh au Panjab, le contrôle des eaux de l'Etat et de l'énergie hydro-électrique qui y est produite, la modification du droit successoral en vue d'éviter le morcellement des terres, un recrutement et des promotions préférentiels dans l'armée, l'attribution au SGPC de la gestion de tous les gurdvara de l'Inde, l'élévation d'Amritsar au rang de ville sainte, la modification de l'article 25 de la constitution qui assimile les Sikhs aux Hindous, la diffusion d'hymnes sikhs depuis le Temple d'Or, l'autorisation pour les Sikhs de conserver leur poignard symbolique à bord des vols intérieurs, l'attribution au panjabi d'un statut de seconde langue pour les Etats voisins à forte population panjabie, l'implantation d'industries lourdes dans le Panjab.

\section{Une société bouleversée}

La campagne d'agitation autour de l'Anandpur Sahib Resolution a pris un tour de plus en plus violent depuis 1981. Ce changement n'est pas sans rapport avec les profondes transformations économiques, démographiques et sociales qui ont affecté le Panjab depuis la «révolution verte» des années 1960-1970 (23).

L'introduction de nouvelles variétés de céréales à haut rendement et d'engrais chimiques, la généralisation des puits tubés à pompe (24) pour assurer en permanence un débit d'irrigation convenable ont alors entraîné un accroissement considérable de la production agricole. En millions de tonnes, la production de blé est passée de 10,24 en 1951 à 79 en 1981; celle du riz de 1,07 en 1951 à 30,56 en 1981 (25). $80 \%$ des terres arables sont désormais irriguées, et l'on fait deux récoltes par an sur $65 \%$ d'entre elles (26). Le ren-

22-Le texte intégral se trouve dans Kuldip Nayar, et Khushwant Singh,op. cit., pp. 135sq. Sur les revendications des Sikhs, cf. également A.-S. Narang, op. cit., pp. $218 \mathrm{sq}$.

23-Il existe une abondante bibliographie sur la révolution verte dans le Panjab. Les principaux aspects de cette révolution, ainsi que les questions en débat à son sujet, sont présentées dans A.-S. Narang, op. cit., pp. 22 sq.

24-Entre 1965-66 et 1978-79, la consommation d'engrais par acre est passée de $8,84 \mathrm{~kg}$ à $90,96 \mathrm{~kg}$ dans le Panjab (de 4,90 kg à 29,80 kg en Inde, Panjab inclus). En 1972, il y avait dans le Panjab un puits tubé à pompe pour 14 ha de terres ensemencées, contre un pour 43 ha en Inde (cf. Kuldip Nayar et Khushwant Singh, op. cit., p. 132). 25-Cf. A.-S. Narang, op. cit., p. 23. 
dement atteint 3 à 5 tonnes de blé ou de riz à l'hectare (27). Le nombre des tracteurs est passé de 50000 en 1975 à 100000 en 1981 (28). L'agriculture représente $60 \%$ du revenu de l'Etat et donne du travail à $62,7 \%$ de sa population active (29). Le Panjab fournit désormais $63 \%$ du blé et $56 \%$ du riz acheté par le Central Pool (30).

Ce tournant vers une agriculture hautement intensive a globalement autant profité aux agriculteurs jat qu'aux commerçants hindous des villes. Le revenu annuel par tête des Panjabis est de loin le pluș élevé de l'Inde. Mais ce développement a accentué les inégalités (31). Le pourcentage des agriculteurs propriétaires de leurs terres (dont $11 \%$ sont louées à des métayers (32)) et celui des ouvriers agricoles sont passés respectivement de $82,7 \%$ et $17,3 \%$ en 1961 , à $68 \%$ et $32 \%$ en 1971 et à $61,74 \%$ et $38,26 \%$ en 1981 . Les inégalités sont grandes entre les propriétaires eux-mêmes. $10 \%$ d'entre eux seulement possèdent plus de 20 acres de terres, $52 \%$ de 2 à 20 acres et $38 \%$ moins de 2 acres (33). Semblablement, 30,6\% des familles panjabies (mais $38 \%$ des familles rurales pour $10,8 \%$ des familles urbaines) ont un revenu mensuel inférieur ou égal à 300 roupies, et $8,8 \%$ un revenu mensuel compris entre 301 et 1000 roupies (34).

Le rapide développement économique du Panjab a eu également d'importantes conséquences démographiques (cf. tableau 2). Faute de trouver

Tableau 2

Evolution de la population du Panjab

\begin{tabular}{lr|r|r|r|r|r} 
& 1881 & 1911 & 1941 & 1951 & 1971 & 1981 \\
\cline { 2 - 7 } Musulmans & 47,56 & 51,06 & 52,88 & & & \\
Hindous & 43,84 & 35,79 & 29,79 & $\cong 67$ & 37,54 & $\cong 45$ \\
Sikhs & 8,22 & 12,11 & 14,62 & $\cong 30$ & $\cong 60$ & $\cong 52$ \\
autres & 0,38 & 1,04 & 2,71 & 3 & 4,26 & 3 \\
total & 100 & 100 & 100 & 100 & 100 & 100
\end{tabular}

Sources : P. Brass, op. cit., p. 299 ;

A. S. Narang, op. cit., pp. 145 sq.; G. Etienne, op. cit. A partir de 1951, les chiffres concernent le seul Panjab indien.

du travail ou d'avoir des revenus suffisants, nombre de Sikhs ont émigré, au moins temporairement, vers l'Europe, l'Amérique du Nord et le MoyenOrient. Dans le même temps l'expansion agricole a attiré vers le Panjab des populations hindoues originaires des régions défavorisées de la moyenne

26-Cf. Avtar Singh Malhotra, Au nom de la fraternité des simples gens, Nouvelle revue internationale, 306, février 1984 pp. 118 sq.

27-D'après G. Etienne, Le rôle politique et économique des Panjabis en Inde et au Pákistan, conférence prononcée au Centre d'études de l'Inde et de l'Asie du Sud, Maison des sciences de l'homme, le 4 février 1984.

28-Cf. Avtar Singh Malhotra, art. cit., p. 118.

29-Cf. A.-S. Narang, op. cit., p. 22.

30-Cf. G. Etienne, loc. cit.

31-En dépit de l'avis contraire émis par certains, tels G.-S. Bhalla (The Green Revolution in the Punjab, New Delhi, Centre for the Study of Regional Development, Jawaharlal Nehru University, s.d).

32-Cf. A --S. Narang, op. cit., p. 26.

33-Cf. Avtar Singh Malhotra, art. cit., p. 119.

34-6f. A.-S. Narang, op. cit., p. 26.

\section{"Qu'est-ce que vous faites là?»*}

Nous savons que le 31 octobre au matin, quand la première balle fut tirée sur Indira $J i$, elle fut si surprise qu'elle laissa échapper: "Qu'est-ce que vous faites là ?».

Quelle grande confiance. En la bonté de l'homme: alors même qu'elle voyait le mal partout, elle ne laissait pas d'être surprise, et au moment où une balle venait de l'atteindre, elle laissa échapper : "Qu'est-ce que vous faites là ?». Un tel acte a été commis au nom d'une idée horrible, certes, mais se venger procède d'une idée tout aussi horrible (1)...

Aucune blessure ne soigne jamais une blessure... La souffrance est à la portée de l'entendement, mais meurtres et massacres sont hors de portée de la souffrance. Et voici que de telles atrocités se propagent dans tout le pays, comme si celle au nom de qui ces horreurs sont commises n'avait pas dit : "Qu'est-ce ce que vous faites là ?». $J$ 'y songe - quand le pays commettra une erreur, quelle qu'elle soit, où que ce soit, de quelque façon que ce soit, s'il lui est possible d'entendre, la voix d'Indira toujours lui dira : "Qu'est-ce que vous faites là ?».

Indira n'est pas le nom d'un individu, c'est le nom de la conscience de notre pays.

* Texte d'Amrita Pritam, Nagmani, 224, décembre 1984, p. 2 Amrita Pritam, éditrice de Nagmani et écrivain vivant le plus célèbre du Panjab, était une amie personnelle d'Indira Gandhi.

1-Allusion aux massacres de Sikhs qui ont suivi l'assassinat d'Indira Gandhi, cf. India Today, 30 novembre 1984, pp. 26sq. 


\section{"Ce sang vous dit»*}

Ouf !

Qu'est-il arrivé,

comme si ce qui devait arriver était arrivé.

La lampe du Panjab était une rose.

Il y avait besoin du sang pur

d'un sacrifice.

A vec l'allumette du courage,

il alluma la lampe de la paix.

Il se conforma

à l'Adi Granth.

Il suivit la trace

de Guru Teg Bahadar (1).

Il alla à Delhi,

vers l'est,

vers le soleil.

La tolérance de Guru Nanak (2),

la volonté de Guru Arjan (3),

à la douceur de laquelle obéir apporte la paix,

la ténacité de Teg Bahadar,

l'héroisme de Gobind Singh (4),

les vers de Farid (5),

les hymnes de Kabir (6),

les psaumes de Mira Baï (7)

l'accompagnaient.

Il revint dans le Panjab.

Pour atteindre ainsi la lune et le soleil.

il fallait qu'il vînt en vainqueur.

L'épée que Sri Guru Gobind Singh

mit à la ceinture de l'Inde entière,

il en défendit l'honneur:

tel un bouclier pour l'Inde,

il préserva le cordon sacré et le tikka (8).

Comme le rabab de Mardana (9),

il disait doucement :

"Laboureurs, passez la charrue,

commerçants, ouvrez vos boutiques!».

Tel son gourou,

il réunissait ceux qui étaient séparés,

il réparait ce qui était brisé,

il vénérait le temple (10)

et hissait toujours plus haut

le drapeau doré du gurdvara.

A vec l'allumette du courage,

il a allumé la lampe de la paix.

Une pluie de balles

dans le gurdvara de Sherpur (11).

Mais combien y a-t-il de fous,

de menteurs et de trompeurs?

La lune et soleil

peuvent-ils mourir aussi?

Les lampes allumées

par les hymnes des gourous

peuvent-elles s'éteindre?

Voyez comme resplendissent

les vaillants Ghadrites,

Bhagat Singh et tant d'autres.

Quand on eut mis

Jésus en croix et perrcé de clous

son beau corps,

quand son sang coula

comme des roses rouges,

des cieux les anges

prirent des coupes et vinrent

recueillir son sang,

qu'il ne se perdit pas.

Vaillants Panjabis,

prenez des coupes et venez

recueillir ce sang.

et que ce soient les coupes

de coeurs purs et irréprochables.

Ce sang vous dit :

«Il n'est ni ennemi ni étranger...

Les hommes sont tous de la même caste».

* Cet éloge funèbre du dirigeant akali Sant Singh Longowal est l'oeuvre d'un écrivain communiste, Santokh Singh Dhir ; il a été publié dans Arsi, septembre 1985, p. 4. Santokh Singh Dhir écrivit après l'assaut du Temple d'Or : "C'est un fardeau/D'être un Sikh de Guru Nanak,/D'être un soldat de Lénine». Cf. Santokh Singh Dhir, Samman (Fardeau), Arsi, août 1984, p. 6.

1 -Neuvième gourou des Sikhs.

2-Premier gourou, fondateur du sikhisme.

3 -Cinquième gourou.

4-Dixième et dernier gourou.

5-Soufi panjabi (mort ca. 1575) dont certains couplets sont inclus dans l'Adi Granth (cf. Lajwanti Rama Krishna, op. cit., pp. 1sq.). Le point de vue qui attribue ces couplets au grand saint cisti Baba Farid Ganj-i Shakar mort en 1265 (cf. Surinder Singh Kohli, $A$ Critical Study of the Adi Granth, Delhi, Mobilal Banarsidass, 1961) est erroné, la langue du Farid de l'Adi Granth ne pouvant remonter au 13 e siècle.

6-Célèbre poète de la tradition sant de l'Inde du Nord qui vécut au début du $15 \mathrm{e}$ siècle et dont de nombreux textes sont inclus dans 1'Adi Granth (cf. C. Vaudeville, Kabir Granthavali (Doha), Pondichéry, Institut français d'indologie, 1957 ; Idem, Au Cabaret de l'Amour, Paris, Gallimard, 1959 ; Idem, Kabir, t. 1, Oxford, Oxford University Press, 1974 ; W.-H. Mc Leod, Guru Nanak and the Sikh religion, op. cit., pp. $151 \mathrm{sq}$.; R. Mc Gregor, Hindi Literature from its Beginnings to the 19th Century, in J. Gonda (ed.), A History of Indian Literature, VIII, 6, Wiesbaden, Harrassowitz, 1984, pp. 46sq.

7 -Poétesse hindie du 16e siècle, auteur de poèmes dévotionnels à Krishna (cf. N. Balbir, Chants mystiques de Mirabai, Paris, 1979 ; R. Mc Gregor, op. cit., pp. 80sq).

8 -Cette représentation des Sikhs, valeureux paysans combattants, défenseurs des Hindous, a été propagée par les écrits du plus célèbre écrivain de la Singh Sabha, Bhaï Vir Singh (cf. D. Matringe, L'apparition de la nouvelle..., art. cit.). Le tikka est la marque portée sur le front par les Hindous.

9-Musicien musulman de caste Dum (sur les Dum, cf. D. Ibbetson, Punjab Castes, Lahore, 1882, réimpr. Lahore, Mubarik Ali, Ltd, 1974 , pp. 175sq.), disciple fidèle et compagnon de voyage de Guru Nanak (cf. W.-H. Mc Leod, Guru Nanak, op, cit., pp. 37sq. ; W. OwenCole, op. cit., pp. 1sq.).

10 -Cést-à-dire le temple hindou ; allusion à la tolérance de Longowal.

11-Sur l'assassinat de Longowal à Sherpur, cf. India Today, 15 septembre 1985 vallée du Gange (Uttar Pradesh oriental et Bihar principalement). Alors que sa journée de travail était payée à un journalier 2 à 3 roupies dans le Bihar en 1978, elle lui était payée 12 à 14 roupies dans le Panjab. Le nombre des travailleurs saisonniers qui se rendent chaque année dans le Panjab est estimé à $400000 ; 100000$ s'y établissent définitivement (35). Ce double phénomène migratoire est lourd de conséquences sur l'équilibre linguistique et communautaire de l'Etat. Alors qu'en 1971, $60 \%$ des Panjabis étaient sikhs et $37,54 \%$ hindous, la proportion des Sikhs était tombée à $52 \%$ en 1981, dont au moins $20 \%$ de mazhabis, descendants d'intouchables convertis et pour la plupart ouvriers agricoles (36). Or ces derniers. votent systématiquement contre l'Akali Dal, parti de leurs patrons, répartissant équitablement leurs voix entre les Partis communistes, par

36-Cf. A.-S. Narang, op. cit., p. 20 et G. Etienne, loc, cit. 
espoir d'émancipation, et le Congrès, pour la relative protection «sociale démocrate» qu'il est susceptible de leur accorder (37). Ceux d'entre eux qui votent pour le Congrès rejoignent sur ce point la grande bourgeoisie panjabie des affaires et du commerce, essentiellement hindoue, et dont les intérêts, parfaitement intégrés à l'économie indienne, sont très bien servis par le Congrès et le gouvernement central.

Les changements démographiques ont donc une incidence politique immédiate. En réduisant la proportion des gros et moyens propriétaires fonciers sikhs, les migrations et la concentration des terres ont effrité la base sociale de l'Akali Dal (38). Le parti, qui a toujours été devancé par le Congrès aux élections locales, n'a jamais pu exercer le pouvoir qu'en concluant des alliances, en 1967 avec les Partis communistes, le Jan Sangh (droite hindoue modérée), le Republican Party et les Indépendants (39), et en 1977, avec la coalition Janata (droite hindoue, animée par le Jana Sangh) (40).

Une telle situation amena la direction modérée du parti à envisager de transformer l'Akali Dal en un grand parti d'opposition régionale (41), comme il en existait ailleurs en Inde, à la périphérie de la ceinture hindie. La laïcisation devait permettre au parti de se maintenir au pouvoir en diminuant les risques de conflit avec les Hindous. Ses dirigeants s'attachèrent donc à mettre en avant les revendications susceptibles de concerner tous les Panjabis. L'Akali Dal déplore en premier lieu que l'industrie, surtout l'industrie lourde, soit peu développée dans le Panjab, dont elle ne représente que $12 \%$ du revenu (alors qu'elle compte pour 22,6\% dans celui de l'Union dans son ensemble) (42). Dans sa propagande, il insiste sur le fait que, des 3,6 milliards de roupies déposés en moyenne chaque année dans les banques du Panjab depuis 20 ans, 2,5 ont été utilisés hors du Panjab au lieu de servir au développement industriel de l'Etat. Ainsi, $70 \%$ du coton et $60 \%$ de la mélasse produits dans le Panjab sont traités hors de ses frontières (43). Dans le même temps, une proportion élevée des eaux du Panjab et de l'énergie hydro-électrique produite dans l'Etat sont détournées par le gouvernement central, qui en a le contrôle, vers le Haryana et le Rajasthan (44). Les agriculteurs, sikhs dans leur grande maiorité, se voient contraints de recourir massivement à l'utilisation de puits tubés à pompe diesel ou

37-Cf. A.-S. Narang, op. cit., p. 189.

38-Ibid., pp. 179 et 194 sq.

39-Ibid., p. 179.

40-Ibid., pp. 193sq. La situation créée par les élections du 25 septembre 1985 est donc inédite puisque l'Akali Dal disposera de la majorité absolue des sièges dans la nouvelle assemblée : 73 sièges sur 115 , contre 32 au Congrès, 4 au Bharatya Janata Party, 1 à la coalition communiste et 4 aux Indépendants, cf. India Today, 15 octobre 1985, pp. 26sq.

41-Cf. A.-S. Narang, op. cit., p. 204.

42 -Ibid., p. 22.

43-Ibid., p. 219.

44-Cf. J. Pettigrew, Take not Arms against thy Sovereign the Present Punjab Crisis and the Storming of the Golden Temple, South Asia Research, IV, 2, novembre 1984, pp.102sq. électrique, dont le coût d'exploitation moyen s'élève à 1200 roupies par acre et par an ; le coût de production des céréales dépasse parfois le Procurement 'Price fixé par'le gouvernement central (45). Il en résulte, chez les paysans sikhs, un profond malaise et un grand mécontentement, particulièrement sensible dans la ieunesse, et qu'ont su exploiter les radicaux de l'Akali Dal et les extrémistes.

L'écrivain-journaliste sikh Khushwant Singh faisait remarquer dans un séminaire organisé récemment à New-Delhi sur le terrorisme dans le Panjab : «Les terroristes du Panjab sont des jeunes gens éduqués, issus des couches moyennes. I1 s'agit de ieunes ruraux sans emploi et 'looking for something to do'» (46). En effet, ces ieunes Sikhs, conscients de leur histoire et d'une identité qu'ils cherchent à affirmer, ne sont plus intéressés par le travail à la campagne, sur des terres sans cesse plus morcelées et pour un profit réduit. Ils ne trouvent guère de débouchés dans le commerce et l'industrie, dominés par les Hindous, et en ont de moins en moins dans l'armée. Le recrutement des Panjabis et, partant, des Sikhs, y est en effet en baisse constante. Les Sikhs ne formaient plus que $12 \%$ de l'armée indienne en 1981, contre $33 \%$ aux derniers jours du Raj. Mais leur nombre est appelé à baisser encore, la part du Panjab ayant été fixée, proportionnellement à sa population, à 2,5\% de l'effectif de l'armée indienne, soit 1,3\% pour les Sikhs (47). Autant de faits qui ont permis à la ligne dure de l'Akali Dal de dénoncer les modérés au nom des intérêts supérieurs de la «nation sikhe» (sikh qaum) (48). Selon ses tenants, en effet, l'exercice du pouvoir politique par l'Akali Dal doit avoir pour fin première la protection du sikhisme contre la discrimination dont il serait l'objet de la part du gouvernement central et du Congrès. A preuve, selon eux, les faveurs accordées au Haryana et au Rajasthan hindous, au détriment du Panjab, patrie des Sikhs.

Dans son ambiguïté fondamentale, l'Anandpur Sahir Resolution reflète fidèlement la dualité de l'Akali Dal : s'y mêlent à des revendications économiques et politiques propres à tous les Panjabis des revendications religieuses particulières aux Sikhs. Après le triomphe du Congrès aux élections de 1980, c'est finalement au nom de la lutte contre la discrimination que l'Akali Dal, rejeté dans l'opposition, a remis en avant sa doctrine de l'inséparabilité de la religion et de la politique et lancé ses morca (campagnes d'agitation) autour de l'Anandpur Sahib Resolution (49).

$\overline{45-\text { Ibid. Nombre de petits exploitants ont même été }}$ contraints de s'endetter.

46-Growth of Extremism in the Punjab : Cause and Cure, National Seminar organized by the Panchnad Research Institute, compte rendu in Overseas Hindustan Times, 31 aout 1985 , p. 5 , sous le titre : Concerted Efforts must for Normalcy in the Punjab.

47-Cf. A.-S. Narang, op. cit., p. 220 et J. Pettigrew, art. cit., p. 109.

48-Sur l'opposition radicaux/modérés dans l'Akali Dal au cours de la période récente, cf. A.-S. Narang, op. cit., pp. 213 sq.

49-Cf. A.-S. Narang, op. cit., pp, 226sq. 


\section{Les partis en présence}

Ce recours à une argumentation «communaliste» ne pouvait que favoriser la montée d'un extrémisme fondamentaliste sikh, symbolisé par le passage de Sant Jarnail Singh Bhindranwale de l'état de simple prédicateur de village au statut de dirigeant charismatique. De ce courant extrémiste, aussi bien l'Akali Dal que le Congrès ont dû depuis tenir compte.

Les partisans d'un Etat sikh indépendant, le Khalistan, issus de la branche radicale de l'Akali Dal, s'organisèrent en 1978 dans la Dal Khalsa (Armée des Purs) (50). Il s'agissait à l'époque d'une douzaine d'extrémistes, regroupés autour de Kapur Singh, ancien Indian Civil Service Officer, et du Dr. Jagjit Singh Chauhan (51). Leur nombre était encore estimé à une soixantaine en 1981 (52), l'année où certains d'entre eux détournèrent un avion des Indian Airlines vers Lahore. Mais depuis, les organisations terroristes ont proliféré : Babbar Khalsa; branche dure de l'All India Sikh Students Federation ; Akhnand Kirtani Jatha ; anciens partisans de Bhindranwale, regroupés dans l'United Akali Dal, désormais dirigé par Baba Joginder Singh, le père de Bhindranwale, etc. (53). Le nombre d'individus susceptibles de terrorisme serait à présent estimé par les services secrets indiens à 2000 ou 3000 (54).

Il est une organisation beaucoup plus mystérieuse et autrement redoutable : le Dashmesh Regiment (Dixième régiment, par allusion au dixième gourou sikh). Le Regiment serait organisé autour de militaires sikhs à la retraite dans les campagnes. Il semblerait avoir réussi à infiltrer l'armée et la police et jouer un grand rôle tant dans l'exécution des attentats que dans l'entraînement des jeunes terroristes.

Quant à Bhindranwale, sa percée fut en partie due aux manoeuvres du Congrès pour encourager les rivalités à l'intérieur de l'Akali Dal entre le courant modéré de son président, Harchand Singh Longowal, et sa frange radicale, animée par Jagdev Singh Talwandi. Elle répondait aussi à l'enthousiasme que sa défense agressive des «droits des Sikhs» soulevait chez les jeunes Sikhs de la petite et moyenne paysannerie dont la situation, comme il a été dit, ne cessait de se dégrader.

Tout remonte à 1978 (55). Cette année-là, Bhindranwale prit la tête d'une expédition contre la secte dissidente des Nirankaris. Il s'en suivit d'autres confrontations meurtrières qui culminèrent avec l'assassinat du gourou nirankari à Delhi en 1980. Bhindranwale, soupçonné d'être impliqué, fụt arrêté. Mais son combat l'avait rendu si popu-

\section{0-Ibid., pp. 221sq.}

51-Ce dernier fut discrédité par sa présence au Pakistan, où ses propositions trouvaient un écho favorable, durant la guerre indo-pakistanaise de 1971, cf. C. Shackle, The Sikhs, The Minority Rights Group, Report 65, Londres, 1984, p. 11.

52-Cf. A. S. Narang, op. cit., p. 222.

53-Cf. Kuldip Nayar et Khushwant Singh, op. cit., p. 88. Sur l'affaiblissement récent de l'United Akali Dal, cf. Overseas Hindustan Times, 23 novembre 1985, p. 1.

54-J.-P. Dardaud, L'Inde aux prises avec l'extrémisme sikh, Le Monde diplomatique, octobre 1985, pp. 1, 20sq. laire que de vastes manifestations furent organisées, auxquelles l'Akali Dal dut prendre part. Bhindranwale fut relâché et devint alors le héros des jeunes gens de 1'All India Sikh Students Federation. Le cylce de la violence était engagé. Bhindranwale fut arrêté une nouvelle fois en 1981, après l'assassinat de Lala Jagat Narain, directeur d'un journal prohindou. Mais sa relaxe rapide accrut encore son prestige et le gouvernement local se montra incapable de mettre un terme aux actions violentes de ses partisans. En 1982, Bhindranwale s'installa triomphalement dans le Guru Nanak Niwas, un hôtel situé dans l'enceinte du Temple d'Or. Le nombre de ses fidèles se multiplia et Bhindranwale attira dès lors l'attention de tous les observateurs du drame panjabi, parmi les acteurs duquel figuraient en bonne place ses escadrons de choc à motocyclette. La situation ne cessa de se dégrader tout au long de l'année 1983, perturbant gravement la vie économique du Panjab et entraînant des opérations de vengeance de la part d'organisations extrémistes hindoues dans le Harayna et ailleurs. Le remplacement du gouvernement local par la President's rule en octobre ne changea rien au cours des événements. Bhindranwale s'installa au coeur du Temple d'Or, dans l'Akal Takht construit par Guru Hargobind, le siège suprême de l'orthodoxie sikhe. C'est là qu'il fut tué au combat lors de l'assaut donné au Temple d'Or par l'armée indienne en juin 1984, assaut qui fit 200 morts parmi les soldats et 1000 parmi les militants sikhs et causa la destruction de l'irremplaçable Sikh Research Library ainsi que celle du local et des archives du SGPC.

Pour enflammer leurs sentiments religieux et inciter les Sikhs à ne pas se contenter de concessions limitées de la part du gouvernement central, Bhindranwale et les siens avaient recours à toute une mythologie fondée sur l'histoire des Sikhs. Menacés par les Musulmans, les Sikhs du temps jadis avaient dû s'armer sous les gourous Hargobind et Gobind Singh. Le $18 \mathrm{e}$ siècle avait été celui où la lutte armée contre les oppresseurs avait permis aux Sikhs de fonder leur Etat souverain dans le Panjab. Pour Bhindranwale et ses partisans, le même processus était en oeuvre. La reprise de l'administration des gurdvara aux mahant, l'expulsion des colonisateurs britanniques et l'obtention de la Panjabi Suba en 1966 étaient les premières étapes d'une marche vers la patrie des Sikhs pour laquelle ils livraient les dernières batailles et où la formule gravée sur le frontispice du Temple d'Or prendrait tout son sens : raj karega khalsa (la Khalsa régnera)

S'adressant aux familles dont les fils étaient morts dans de vrais ou prétendus accrochages avec la police, aux Sikhs orthodoxes, aux paysans mécontents qui venaient de très loin pour l'écouter et à toute une jeunesse désireuse d'affirmer son identité, Bhindranwale disait (56) : "Nous ne

55-Pour un exposé chronologique commode des événements auxquels je fais allusion dans les pages qui suivent, cf. Kuldip Nayar et Khushwant Singh, op. cit., et aussi, à partir de 1982, la "couverture» des événements par le bimensuel India Today. Sur l'évolution de Bhindranwale, cf. Sunil Sethi, The Great Divide, in Amrajit Kaur et al., op. cit.

56-Cf. J. Pettigrew, art. cit., pp. 102sq. 
sommes pas les disciples d'Indira ni de son père, mais les Sikhs de Guru Hargobind, maître du spirituel et du temporel, et du vénéré Guru Gobind Singh» (c'est-à-dire des deux gourous auxquels est attribuée la militarisation du Panth).

$\mathrm{Ou}$ encore, quelque temps avant l'opération Blue Star : "Vous ne pouvez obtenir justice que s'il y a une loi, quelqu'un pour la faire appliquer et un droit d'appel. Où voulez-vous aller demander justice quand personne n'a cure du juge ni de la loi et que la police est toute-puissante ? Vous devrez résoudre vos problèmes de vos propres mains. Ils commettent envers nous des atrocités, exterminent notre jeunesse, brûlent notre Livre Saint et insultent nos turbans. Quand il en est ainsi, à quoi bon porter plainte ? Vous n'avez pas besoin d'une autorisation de port d'armes. Guru Hargobind n'en sollicita pas une de Jahangir ni Guru Gobind d'Aurangzeb. Quand les atrocités imposées à un peuple paisible dépassent les bornes, sa jeunesse tend à suivre l'exemple de Guru Hargobind. Après tout, quand il n'y avait plus ni loi, ni preuve, ni appel, les Sikhs de la Khalsa ont suivi ce chemin. En agissant comme il le fait, le gouvernement risque de compromettre le futur. Etre en paix, est-ce s'asseoir sur une plaque de fer rougie ? Etre en paix, est-ce être décapité dans une cage ? (57). Mais quand notre foi même est la proie des flammes, que peut-il y avoir de pire? De même que pour un musulman il n'y a rien au-delà de La Mecque, de même il n'y a rien pour nous au-delà du Sri Harimandir Sahib (le Temple d'Or). Nous n'attaquerons la maison ni ne pillerons la boutique de personne. Mais si un homme enivré de pouvoir s'en prend à nous, nous répondrons. Nous ne sommes pas des femmes porteuses de bracelets. Le Donneur-de-ses-fils (Guru Gobind) commanda aux Sikhs de se battre à un contre 125000 ennemis. Nos dirigeants actuels nous disent qu'ils vont nous encercler, nous déloger. Nous allons mourir, et par conséquent nous ne pouvons dire qui de la police ou de la sangat (la " communauté» sikhe) lèvera nos corps. Mais je voudrais dire aux Sikhs des forces de police, s'il vous reste un tant soit peu d'honneur et de fierté, si vous croyez au sikhisme, si vous êtes dignes d'être appelés les fils du Gourou (le Dieu des Sikhs), alors ne marchez jamais sur le Hari Mandir».

Face à la propagande des extrémistes, l'Akali Dal a connu une longue période d'hésitation, liée à ses contradictions internes. D'une part il lui fallait éviter d'apparaître comme un parti compromis avec le terrorisme, afin de garder le soutien de sa base rurale aisée et de pouvoir relever le défi du Congrès, soutenu par la bourgeoisie des affaires et du commerce. Mais il lui fallait également iouer des sentiments religieux des Sikhs et du mécontentement de nombre d'entre eux. Ainsi Longowal ,et ses amis reprochaient au Congrès d'appuyer secrètement les extrémistes pour diviser l'Akali Dal et de faire volontairement traîner les négociations pour laisser pourrir la situation. Mais dans le même temps, craignant qu'accepter des concessions religieuses de la part du gouvernement central ne démobilisât une fraction de ses partisans qui s'en serait contentée et ne renforçât les extrémistes, Longowal envoya à la table de négociation, outre trois modérés de sa tendance, deux radicaux. Il ajouta que tout accord devrait être ratifié par l'Akal Takht, institution religieuse suprême du sikhisme (58).

Ce souci affiché de ne pas séparer la religion de la lutte politique permettait à l'Akali Dal, dont la base sociale s'était effritée, de se présenter comme le parti de tous les Sikhs et de garder le contrôle du

57-Allusion à la mort, aux mains des Moghols, de Dayal Das, l'un des disciples favoris de Teg Bahadar et à celle du gourou lui-même, qui fut décapité le lendemain, cf. Hari Ram Gupta, op. cit., pp. 142sq.

58-Cf. A.-S. Narang, op. cit., p. 228.
SGPC, où les extrémistes n'obtinrent pas un siège en 1979 (59). Mais en s'empêtrant dans une rhétorique communaliste, l'Akali Dal se retrouvait «prisonnier de ses propres slogans» (60). Ainsi fut remise en avant la clause de l'Anandpur Sahib Resolution qui exigeait que le Panjab devînt un Etat où les Sikhs eussent la suprématie et pussent librement exprimer leur nationalité, au nom du principe Sikh ikk qaum han (Les Sikhs forment une nation). Une motion votée par le SGPC à l'initiative de son président akali modéré, Tohra,en 1981 allait dans le même sens (61). De tels slogans étaient justement ceux sur lesquels les partisans du Khalistan fondaient leur propagande. Or le parti ne donna de la voix que pour faire refuser à la police le droit de pénétrer dans les lieux de culte pour les fouiller (62), et se refusa toujours, autant par intérêt que par crainte, à condamner clairement les actes terroristes.

L'attitude du Congrès et du gouvernement central n'était pas moins ambiguë. Il s'agissait, pour Indira Gandhi, de discréditer durablement l'Akali Dal afin d'empêcher sa transformation en un parti régionaliste qui pût menacer la domination du Congrès dans le Panjab. Empêcher cette transformation constituait un but commun pour le Congrès, pour les Akali radicaux et pour les extrémistes, ce dont Indira Gandhi tenta apparemment de jouer. Chaque fois que l'Akali Dal, dans les méandres de ses débats internes et avec les extrémistes, sembla prêt à accepter un compromis, le gouvernement central fit la sourde oreille, tout comme il refusa d'associer les partis d'opposition à la recherche d'une solution à la crise panjabie. Ainsi, immédiatement après le lancement par l'Akali Dal d'une campagne d'agitation de masse baptisée Dharam Yudh (Guerre sainte) le 3 août 1982, Prakash Singh Badal, l'un des principaux responsables modérés de l'Akali Dal, informa tous les partis d'opposition que son organisation ne retenait plus que 10 des 45 exigences de l'Anandpur Sahib Resolution (quatre concernant la religion et six concernant tous les Panjabis) et qu'elle était prête à mettre un terme à une morca qui s'annonçait longue et dure si elle obtenait satisfaction (63). Le gouvernement central fit la sourde oreille et rompit par la suite plusieurs fois les pourparlers sur la question de Chandigarh, ou même, une fois, en refusant de changer le nom du rapide Bombay-Amritsar, le Flying Mail, en Golden Temple Express. En 1982, il invita le gouvernement du Haryana à empêcher les Sikhs de se rendre aux Asiades (les jeux olympiques panasiatiques) à Delhi. Même des juges de la Haute Cour et des officiers supérieurs furent soumis à des tracasseries lorsqu'ils voulurent s'y rendre parce qu'ils étaient sikhs. Les morca organisées par l'Akali

59-Cf. Surinder Singh Suri et Narinder Dogra, A Study of the SGPC Elections, March 1979, Punjab Journal of Politics, V. 1, 1981, pp. 114sq.

60-Selon l'expression de Karkisan Singh Surjit, Panjab bare ki sac hai ? (Quelle est la vérité à propos du Panjab ?), Arsi, juillet 1984, pp. 5sq.

61-Cf. A.-S. Narang, op. cit., p. 226.

$62-$ Cf. Harkisan Singh Surjit, art. cit., p. 6.

63-Ibid., p. 8. 
Dal n'avaient jamais tourné à la violence, mais le jour du rasta rokho (bloquez les routes) en avril 1983, la police ouvrit le feu sur ceux qui bloquaient les routes, tuant une vingtaine de personnes. De telles pratiques ne pouvaient que renforcer le sentiment de discrimination éprouvé par de nombreux Sikhs et faire redoubler les violences terroristes.

A plusieurs reprises, des contacts eurent lieu entre le gouvernement central et Bhindranwale qui put faire en 1980 une tournée triomphale à Bombay et Delhi. Tout comme lui, son principal lieutenant fut relâché «sur ordre» après son arrestation. Selon certains, des terroristes qui avaient été signalés à la police auraient été laissés en liberté (64). Enfin, le gouvernement central savait que des armes avaient été accumulées dans le Temple d'Or. Bien qu'il pût invoquer la main de l'étranger et le rôle du Pakistan (65), l'instabilité résultait aussi de sa propre politique et l'agitation profitait, d'une certaine façon, au parti du Congrès. Non seulement elle lui imposait de rester uni dans le Panjab face à des adversaires qu'il cherchait à diviser, mais le sentiment d'insécurité ressenti d'abord par les Hindous, puis leur «soulagement» après l'intervention de l'armée, étaient susceptibles de les faire voter en masse pour le parti gouvernemental aux élections à venir. Du moins est-ce ainsi que l'opposition indienne ressentit la politique menée par Indira Gandhi : lorsqu'il devint évident qu'une action militaire allait ávoir lieu contre le Temple d'Or, les partis de la droite hindoue accusèrent le gouvernement central d'avoir facilité l'armement des terroristes et se montrèrent plus attentifs à l'affirmation par l'Akali Dal que la plupart de ses revendications concernaient tous les Panjabis (66).

64-Ibid.

65-Cf. V.-D. Chopra, loc. cit.

66-Cf. A.-S. Narang, op. cit., p. 230.
Depuis l'assassinat d'Indira Gandhi, la volonté populaire de retour au calme et à l'ordre dans le Panjab et l'attitude conciliante du nouveau premier ministre ont contribué à débloquer la situation. Assuré d'obtenir des concessions majeures, dont la cession de Chandigarh au Panjab, et pressé par sa base, l'Akali Dal a accepté de signer un accord avec le gouvernement central. La propagande du parti consiste désormais à rappeler que les Sikhs ont toujours été proches des Hindous, qu'il y a toujours eu des mariages entre les deux communautés, qu'au 18 e siècle elles étaient unies contre la tyrannie musulmane et qu'elles ont fait cause commune pour libérer l'Inde des colons britanniques et tenter d'éviter la partition. Khushwant Singh citait récemment ce poème Panjabi de Raghbir Singh Bir (67) :

\section{Voici pourquoi nous chérissons l'Inde.}

C'est ici que nos ancêtres ont donné leurs vies.

C'est pour elle que notre dixième gourou a sacrifié ses quatre fils.

Elle est notre Mecque, notre Médine, notre Kaba,

Notre Arabie, notre Karbela,

Notre paradis, notre Royaume-de-la-Vérité

(le suprême paradis sikh)

Et le temple de notre Vrai Gourou (Dieu).

Si, pour sauver l'Inde,

Les Sikhs plus que d'autres ont donné leurs vies,

Ce n'est pas surprenant,

Car personne plus qu'un Sikh

N'est fier d'être indien.

67-Khushwant Singh, Malice towards one and all, Overseas Hindustan Times, 31 aout 1985, p. 7 